\title{
Random walkers based segmentation method for breast thermography
}

\begin{abstract}
In breast thermography diagnostic, proper detection and segmentation of the areola area as well as detection of breast boundaries present the biggest challenge. As the boundaries of breasts especially in the upper quadrants are usually not present, this produces a great deal of challenge to segment breasts automatically. Many approaches have been developed to segment the breast in the past such as Snakes, Active Contours and Circular Hough Transforms, but these methods fail to detect the boundaries of the breast with the required level of accuracy especially the upper boundaries of the breast. By utilizing most recent segmentation method which is Random Walkers, the breast can be segmented accurately which in turn will increase the accuracy and the reliability of computer aided detection/diagnosis systems.
\end{abstract}

Keyword: Image segmentation; Random walkers; Breast thermography; Areola detection 